\title{
SWEET FUTURE OF STEVIA: A MAGICAL SWEETENER
}

\author{
JEEVAN JYOTI ${ }^{1}$, MANINDERJEET KAUR ${ }^{2}$, VIJAY MISHRA ${ }^{2}$, AMIT MITTAL $^{1,3 *}$
}

${ }^{1}$ Department of Quality Assurance, Lovely Institute of Technology (Pharmacy), Lovely Professional University, Phagwara, Punjab, India. ${ }^{2}$ Department of Pharmaceutics, Lovely Institute of Technology (Pharmacy), Lovely Professional University, Phagwara, Punjab, India. ${ }^{3}$ Department of Pharmaceutical Chemistry, Lovely Institute of Technology (Pharmacy), Lovely Professional University, Phagwara, Punjab, India. Email: amit.13145@lpu.co.in

Received: 29 May 2017, Revised and Accepted: 15 November 2017

\section{ABSTRACT}

The plant Stevia rebaudiana is mainly found in tropical and subtropical regions from western North America to South America. This genus is having near about 240 species of shrubs and herbs in the sunflower family (Asteraceae). It exhibits various properties such as antibacterial, antifungal, anti-inflammatory, antimicrobial, antiviral, antiyeast, cardiotonic, diuretic, hypoglycemic, hypotensive tonic, and vasodilator effect. It is an important source of a number of antioxidants, for example, benzoic acid, caffeic acid, chlorogenic acid, ferulic acid, rozmaric acid, protocatechuic acid, salicylic acid, and their derivatives and flavonoids including campherol derivatives, catechin, and its derivatives, epicatechin, luteolin, and its derivatives, rutin, and its derivatives. Day by day, there is remarkable increase in demand of high potency sweeteners. The increasing number of diabetic patients and health conscious individuals would push forward the need for alternatives to sugar. The extract from leaves of Stevia is 200 times sweeter than sugar (glucose, fructose, sucrose, maltose, and lactose). Stevia is a potential alternative source for replacing artificial sweeteners such as saccharin, aspartame, and asulfam.

Keywords: Artificial sweeteners, Stevia, Antioxidant, Flavonoids.

(c) 2018 The Authors. Published by Innovare Academic Sciences Pvt Ltd. This is an open access article under the CC BY license (http://creativecommons. org/licenses/by/4. 0/) DOI: http://dx.doi.org/10.22159/ajpcr.2018.v11i2.20295

\section{INTRODUCTION}

Stevia rebaudiana Bertoni is a perennial herb of significant economic value due to its high content of natural, dietetically valuable sweeteners in its leaves [1,2]. The sweet taste of Stevia is due to diterpene glycoside; it is calorie free and does not metabolize. Therefore, it is established as natural sweetest plant on earth. Stevia possesses many beneficial properties as compared to other sweeteners such as stevia has calorie value $2.7 \mathrm{kcal} / \mathrm{g}$, whereas glucose has calorie value $3.80 \mathrm{kcal} / \mathrm{g}$. Stevia is thermostable and can withstand temperature range of $200^{\circ} \mathrm{C}$. Being non-fermentable, it is used in cooking and baking. It is less expensive as compared to other sweeteners. Stevia is used in the treatment of diabetes and obesity by suppressing appetite and reduces the urge for sweets. Further, it is helpful in the management of weight or to reduce weight [3].

Stevia products are approved in more than 100 countries, and about 5 billion consumers have access to Stevia products (Fig. 1) [4]. The total value of global sweetener market, sugar, high-fructose corn syrup, and non-natural high-intensity sweeteners is about $\$ 70, \$ 60, \$ 7$, and $\$ 1.2$ billion USD, respectively. The global sale of high-purity Stevia extracts in 2013 was about $\$ 150$ million USD and the estimated growth in 20 years is more than $\$ 10$ billion USD [4].

\section{STEVIA CULTIVATION}

A number of countries showing their enthusiasm for its cultivation and for research work. The cultivation of Stevia is mainly done by intensive study and according to its agronomic abilities. Stevia has been considered as a beneficial product due to the high content of sweetness, adaptive nature of the plant in various climates and its medicinal uses. In future, the demand for this beneficial sweetener is relied on progress. The herb is native of the Amambay region, in northeastern Paraguay [5]. The various countries of world which are growing this sweetener are India, Egypt, California, Western Georgia, Italy, Abkhazia, Korea, Slovakia, Czech Republic, Canada, Russia, Indonesia, Brazil, and Argentina [1]. Long-term potential of Stevia leaf production is around 2 million tones showing agricultural industry potential about \$3-4 billion USD [4].

\section{CHEMICAL CONSTITUENTS OF STEVIA}

S. rebaudiana comprise more than 100 chemical constituents, but the most abundant compounds found are steviol glycosides, mainly rebaudioside A and stevioside (Fig. 2) [6].

\section{STEVIOL GLYCOSIDES}

In $S$. rebaudiana, there are more than 30 steviol glycosides with different concentrations of the total steviol glycosides up to $20 \%$ of the dry leaf weight are reported (Tables 1 and 2). The most profound steviol glycosides are stevioside and rebaudioside A, which are present in high amounts [7-17].

\section{NON-GLYCOSIDE DITERPENES (STEREBINS)}

These constituents mainly belong to Labdane-type diterpenes. These compounds were identified using ${ }^{1} \mathrm{H},{ }^{13} \mathrm{C}$ nuclear magnetic resonance (NMR), ultraviolet and infrared spectroscopy, and MS. The low-polarity sterebins do not possess any known pharmaceutical effects. Therefore, the concentration of low-polarity sterebins can be minimized by developing new Stevia lines, and the levels of sweet diterpenes glycosides can be enhanced (Table 3) [18-22].

\section{POLYPHENOLS}

Polyphenols were analyzed as an additional parameter by Folin-Ciocalteu colorimetric method. The total phenolic content obtained was expressed in gallic acid, tannic acid, or catechin equivalents/g or mg of extract or dried leaves. Quantitatively, the phenolic compounds can be analyzed by the use of high-performance liquid chromatography (HPLC) on a C18 column with gradient elution and diode array detection (DAD). The main phenolic compounds analyzed were pyrogallol with $951.27 \mathrm{mg} / 100 \mathrm{~g}$ dry base water extract, 4-methoxybenzoic acid ( $33.80 \mathrm{mg} / 100 \mathrm{~g})$, p-coumaric acid (30.47 mg/100 g), 4-methylcatechol (25.61 mg/100 g), and sinapic 




Fig. 1: Number of products launched by year

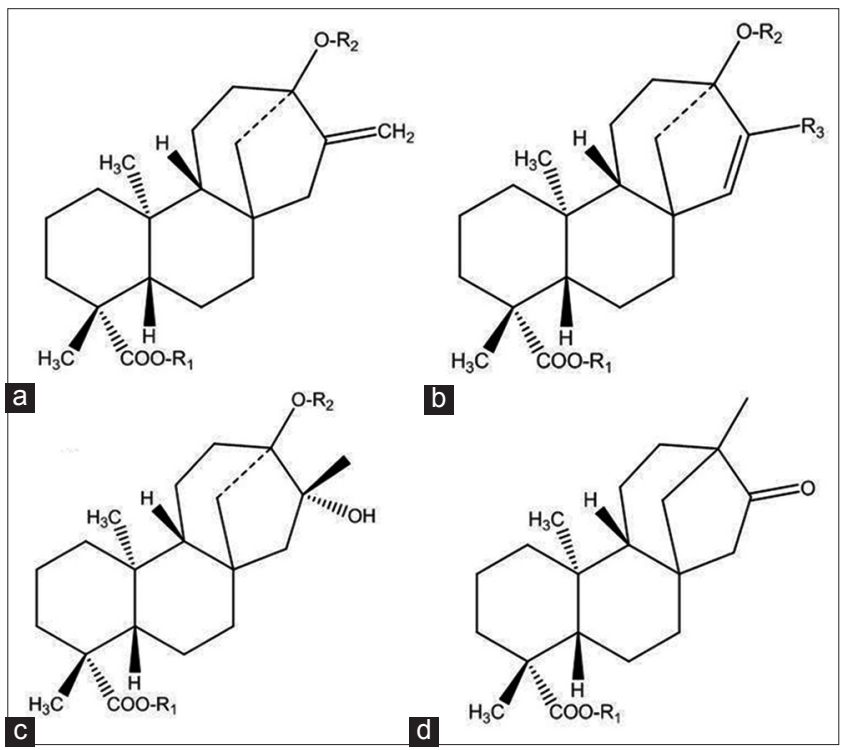

Fig. 2:(a-d) Different ent-kaurene body structures of steviol glycosides

and cinnamic acid (Fig. 3). Various chlorogenic acid and other phenolic compounds found in S. rebaudiana are enlisted in Table $4[23,24]$.

\section{FLAVONOIDS}

In leaves of Stevia, the observed flavonoids are concerned with subgroups of flavonols and flavones (Table 5). These were recognized utilizing twodimensional UHPLC-DAD34 and LC-MS/MS and spectroscopic techniques $\left({ }^{1} \mathrm{H},{ }^{13} \mathrm{C}\right.$ NMR, IR, and 2D NMR). Quantitatively, they were detected as total flavonoid content using technique of aluminum chloride colorimetric and the Folin-Ciocalteu assay. Quantitatively, they were broke down as aggregate flavonoid content utilizing an aluminum chloride colorimetric technique and the Folin-Ciocalteu measure [25-28].

\section{POLYHYDROXY INDOZILIDINE ALKALOID}

The steviamine an indozilidine iminosugar alkaloid (Fig. 4) was extracted from leaves of stevia plant. Alkaloids of this type belong to Hyacinthaceae family, but never found in Asteraceae. Different pharmacological and biomedical properties have been reported in iminosugars like the inhibitory effect against glucosidase $[29,30]$.

\section{Natural sweeteners in a human diet [31-34]}

Table 6 shows the natural sweeteners (Sugar alcohols and Other natural sweeteners)

\section{TRADITIONAL MEDICINAL USES OF STEVIA}

Stevia has potential uses such as cardiotonic (strengthens, tones, and balances the heart), sweetener, antimicrobial activities, hypotensive

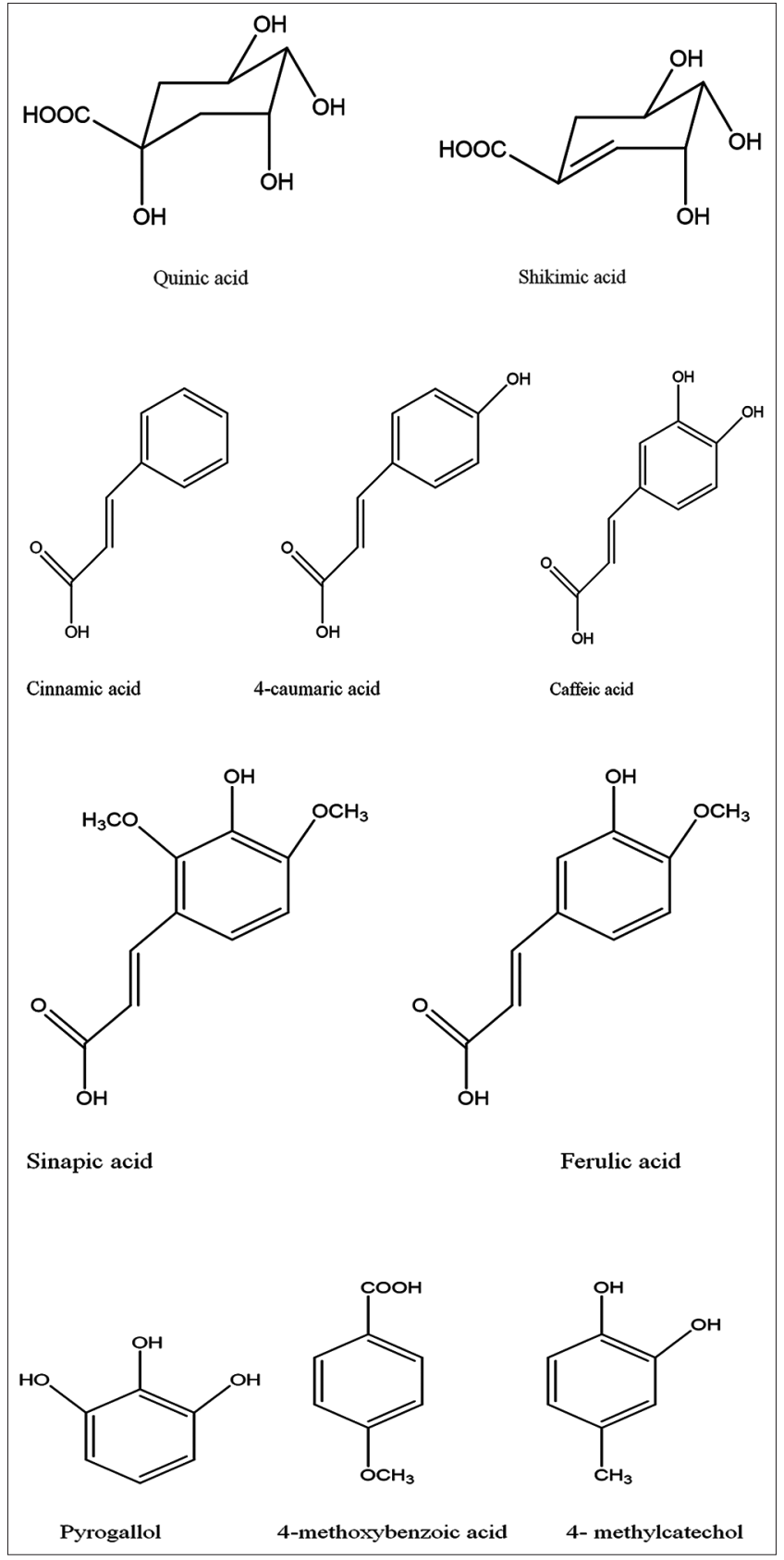

Fig. 3: Substructures of chlorogenic acids

(reduces blood pressure), and hypoglycemic (Table 7) [35]. Due to various natural constituents, stevia is very beneficial for human health.

\section{COMMERCIALIZATION OF STEVIA}

The use of stevia is prohibited for human food because it has not been included in GRAS (Generally Recognized as Safe) status indicated in the documents provided by Dietary Supplement Health and Education Act. On the premise of authentic use and logical proof, Doun Kinghorn of the Herb Research Foundation gave a review for American Herbal Products Association that has proved the safe use of stevia. After this proof, many researchers have studied that stevia possesses a number of medicinal uses and does not have any side effects. As studied by GD Searle and Company, near about 200 reviews stated stevia as "NutraSweet" is safe. Diverse administrative bodies like FDA reassessed the papers and as named new sweetener Neotame is to be promoted by the organization. In the USA, steviol glycoside got the 


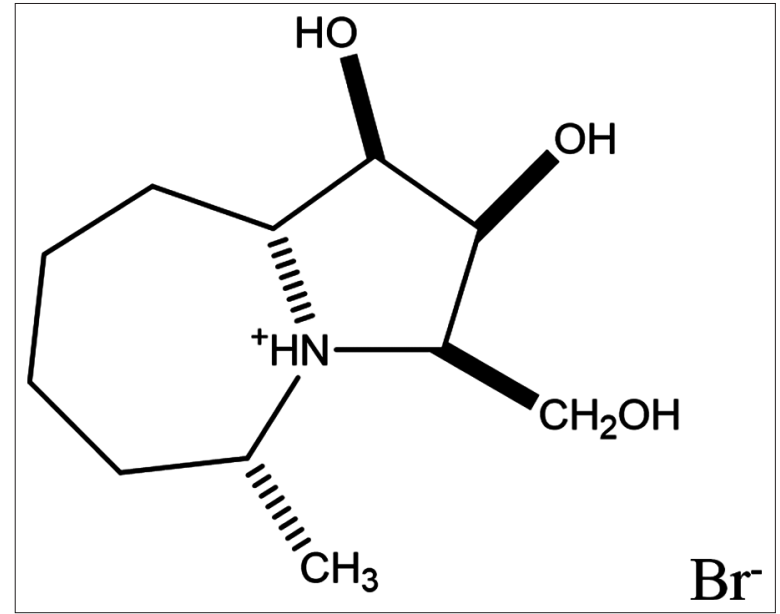

Fig. 4: Structure of steviamine

Table 1: New steviol glycosides

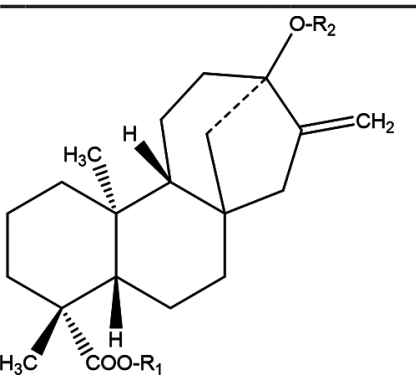

\begin{tabular}{|c|c|c|}
\hline $\begin{array}{l}\text { Steviol } \\
\text { glycosides }\end{array}$ & $\mathrm{R}_{1}$ & $\mathrm{R}_{2}$ \\
\hline Steviolmonoside & $\mathrm{H}$ & Glc $\beta 1-$ \\
\hline Rubusoside & Glc $\beta 1-$ & Glc $\beta 1-$ \\
\hline Steviolbioside & $\mathrm{H}$ & Glc $\beta 1-2 \mathrm{Glc} \beta 1-$ \\
\hline Dulcoside A & Glc $\beta 1-$ & Rha $\alpha 1-2$ Glc $\beta 1-$ \\
\hline Stevioside & Glc $\beta 1-$ & Glc $\beta 1-2 \mathrm{Glc} \beta 1-$ \\
\hline Rebaudioside G & Glc $\beta 1-$ & Glc $\beta 1-3$ Glc $\beta 1-$ \\
\hline Rebaudioside B & $\mathrm{H}$ & Glc $\beta 1-2(\operatorname{Glc} \beta 1-3)$ Glc $\beta 1-$ \\
\hline Dulcoside B & $\mathrm{H}$ & Rha $\alpha 1-2(G l c \beta 1-3)$ Glc $\beta 1-$ \\
\hline Rebaudioside A & Glc $\beta 1-$ & Glc $\beta 1-2(\operatorname{Glc} \beta 1-3)$ Glc $\beta 1-$ \\
\hline Rebaudioside C & Glc $\beta 1-$ & Rha $\alpha 1-2(\operatorname{Glc} \beta 1-3)$ Glc $\beta 1-$ \\
\hline Rebaudioside L & Glc $\beta 1-$ & $\begin{array}{l}\text { Glc } \beta 1-6 \text { Glc } \beta 1-2(\text { Glc } \beta 1-3) \\
\text { Glc } \beta 1-\end{array}$ \\
\hline Rebaudioside $\mathrm{H}$ & Glc $\beta 1-$ & $\begin{array}{l}\text { Glc } \beta 1-3 R h a \alpha \beta 1-2 \\
\text { (Glc } \beta 1-3) \text { Glc } \beta 1-\end{array}$ \\
\hline Rebaudioside F & Glc $\beta 1-$ & Xyl $\beta 1-2(G l c \beta 1-3)$ Glc $\beta 1$ - \\
\hline Rebaudioside E & Glc $\beta 1-2$ Glc $\beta 1$ - & Glc $\beta 1-2$ Glc $\beta 1-$ \\
\hline Rebaudioside D & Glc $\beta 1-2 \mathrm{Glc} \beta 1$ & Glc $\beta 1-2(\operatorname{Glc} \beta 1-3)$ Glc $\beta 1-$ \\
\hline Rebaudioside I & Glc $\beta 1-3$ Glc $\beta 1$ - & Glc $\beta 1-2(\operatorname{Glc} \beta 1-3)$ Glc $\beta 1$ \\
\hline Rebaudioside $\mathrm{K}$ & Glc $\beta 1-2$ Glc $\beta 1-$ & Rha $\alpha 1-2(\operatorname{Glc} \beta 1-3)$ Glc $\beta 1-$ \\
\hline Rebaudioside J & Rha $\alpha 1-2$ Glc $\beta 1-$ & Glc $\beta 1-2(\operatorname{Glc} \beta 1-3)$ Glc $\beta 1-$ \\
\hline Rebaudioside N & $\begin{array}{l}\text { Rha } \alpha 1-2(\text { Glc } \beta 1-3) \\
\text { Glc } \beta 1-\end{array}$ & Glc $\beta 1-2(\operatorname{Glc} \beta 1-3)$ Glc $\beta 1-$ \\
\hline Rebaudioside M & $\begin{array}{l}\text { Glc } \beta 1-2(\operatorname{Glc} \beta 1-3) \\
\text { Glc } \beta 1-\end{array}$ & Glc $\beta 1-2(\operatorname{Glc} \beta 1-3)$ Glc $\beta 1-$ \\
\hline Rebaudioside 0 & $\begin{array}{l}\text { Glc } \beta 1-3 R h a \alpha 1-2 \\
\text { (Glc } \beta 1-3) \text { Glc } \beta 1-\end{array}$ & Glc $\beta 1-2(\operatorname{Glc} \beta 1-3)$ Glc $\beta 1-$ \\
\hline
\end{tabular}

Where Glc: Glucose, Rha: Rhamnose, Xyl: Xylose

GRAS status in 2008 and 2009 [3]. Some of its products in the market of the USA are given in Table 8 [3].

\section{CONCLUSION AND FUTURE PERSPECTIVES}

Stevia is now being used worldwide for its natural sweetening activity and pharmaceutical properties. Some extensive high throughput
Table 2: New steviol glycosides with changes in the ent-kaurene backbone

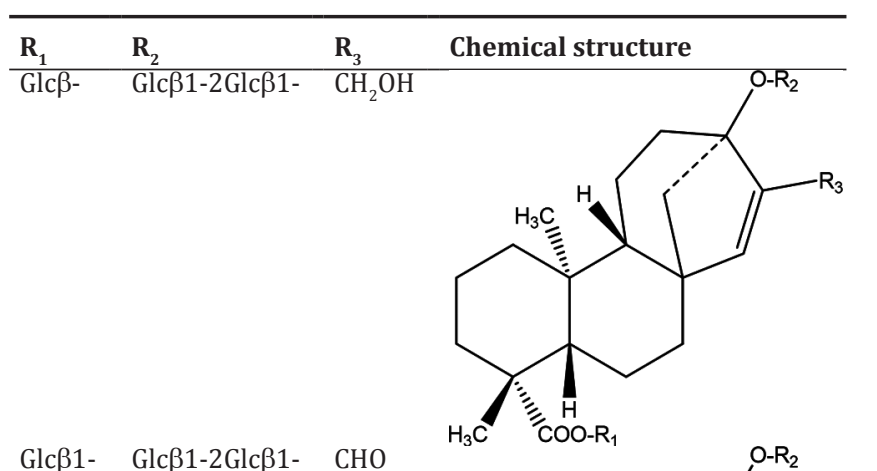

Glc $\beta 1-\quad$ Glc $\beta 1-2 \mathrm{Glc} \beta 1-\quad \mathrm{CH}_{3}$
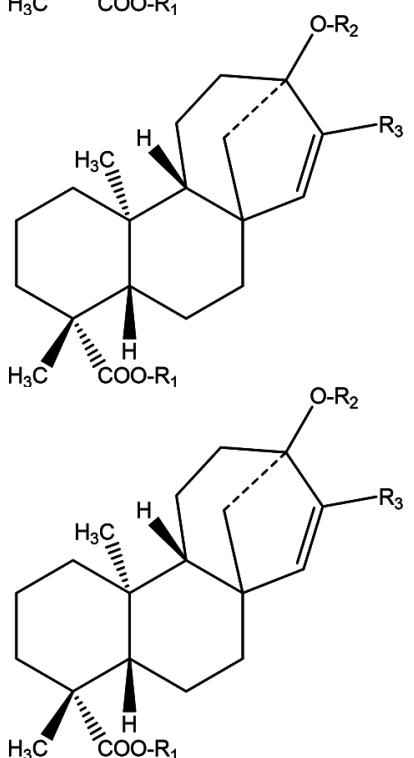

$\mathrm{H}$

Glc $\beta 1-2$

(Glc $\beta 1-3)$

Glc $\beta 1$ -

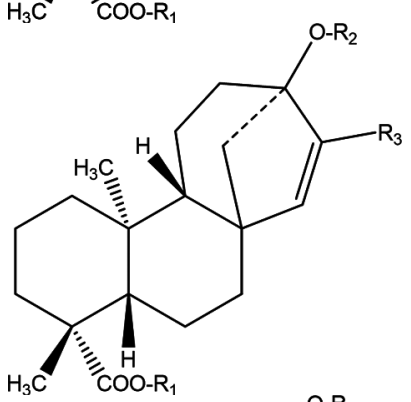

$\mathrm{H} \quad$ Glc $\beta 1-2$

(Glc $\beta 1-3$ )

Glc $\beta 1$ -

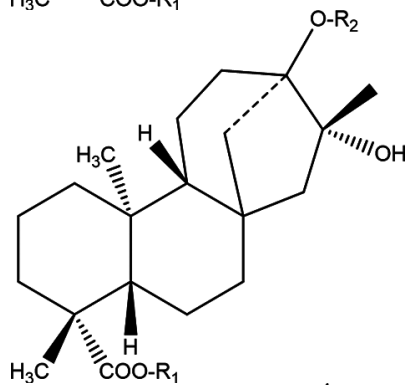

Glc $\beta 1$ -

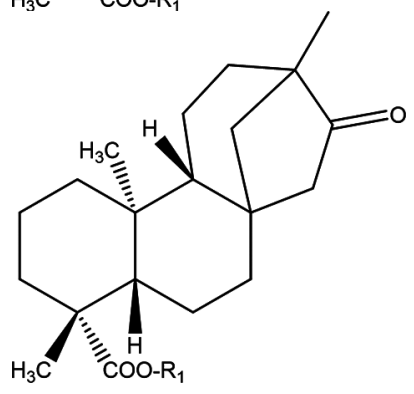




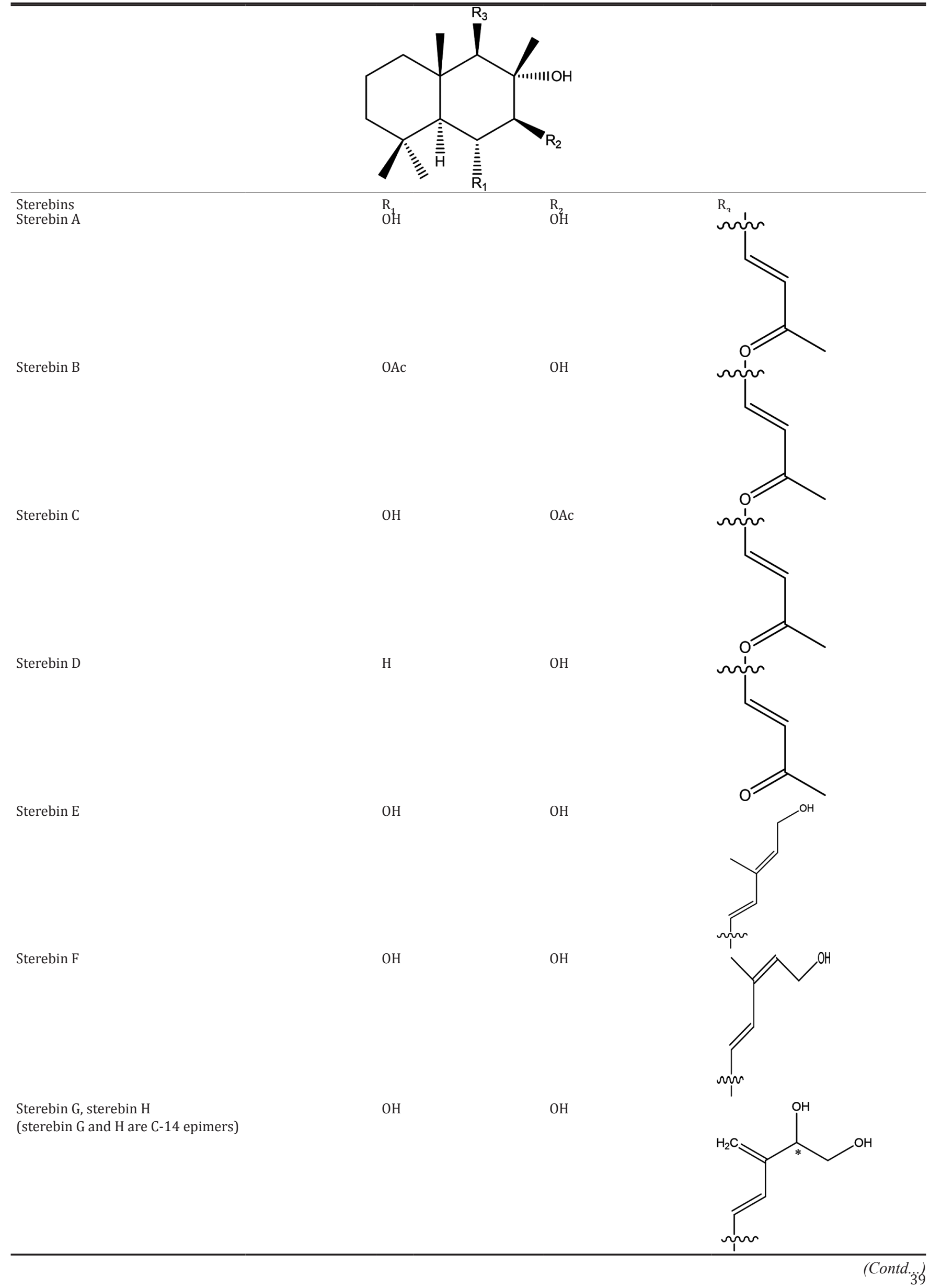


Table 3: (Continued)

\section{$\mathrm{OH}$}

$\mathrm{OH}$

$\mathrm{OH}$

$\mathrm{OH}$<smiles>[Z6]C=CC(=CC=O)CC</smiles>

$\mathrm{O}$<smiles>C=O</smiles><smiles>[Z][Z6](C)=C=CC(=C)C</smiles><smiles>[Z6]C=C/C([Y9])=C\COCOC/C=C(C)\C=C\N</smiles><smiles>[Z][Z](C)=C[C@](C)(O)C=C</smiles>

$\mathrm{HO}$

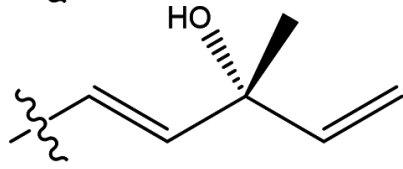

Table 4: List of chlorogenic acid and other phenolic compounds found in Stevia rebaudiana

Name

3-caffeoylquinic acid (3-CQA)

5-caffeoylquinic acid (5-CQA)

4-caffeoylquinic acid (4-CQA)

3,5-dicaffeoylquinic acid (3,5-diCQA)

3,4-dicaffeoylquinic acid (3,4-diCQA)

4,5-dicaffeoylquinic acid (4,5-diCQA)

A cis-3,5-dicaffeoylquinic acid (a cis - 3,5-diCQA)

A cis-4,5-dicaffeoylquinic acid (cis -4,5-diCQA)

Cis-4,5-dicaffeoylquinic acid ( cis -4,5-diCQA)

A cis-4,5-dicaffeoylquinic acid (a cis -4,5-diCQA)

5-p-coumaroylquinic acid (5-p-CoQA)

Caffeoyl-feruloylquinic acid (CFQA)

4-Caffeoyl-5-feruloylquinic acid (4-C,5FQA)

5-Caffeoylshikimic acid ( 5-CSA)

4-Caffeoylshikimic acid ( 4-CSA)

3-Caffeoylshikimic acid (3-CSA)

5-Feruloylquinic acid (5-FQA)

Feruloylquinic acid ( FQA)

3,4,5-Tricaffeoylquinic acid (3,4,5-triCQA)

1,3,5-Tricaffeoylquinic acid (1,3,5-triCQA)

Tricaffeoylquinic acid ( triCQA)

3,4,5-Tricaffeoylquinic acid ( triCQA)

Pyrogallol

4-Methoxybenzoic acid

4-Coumaric acid

4-Methylcatechol

Sinapic acid

Cinnamic acid 
Table 5: Flavonoids found in leaves of Stevia rebaudiana

\begin{tabular}{|c|c|c|c|c|c|}
\hline Flavonoids & $\mathrm{R}_{1}$ & $\mathrm{R}_{2}$ & $\mathrm{R}_{3}$ & $\mathrm{R}_{4}$ & $\mathrm{R}_{5}$ \\
\hline $\begin{array}{l}\text { Flavonols } \\
\text { Quercetin }\end{array}$ & $\mathrm{OH}$ & $\mathrm{OH}$ & $\mathrm{H}$ & $\mathrm{OH}$ & $\mathrm{OH}$ \\
\hline Quercetin-3-0- $\beta$-D-arabinoside & $\mathrm{OH}$ & $\mathrm{OH}$ & $\mathrm{H}$ & O-arabinoside & $\mathrm{OH}$ \\
\hline Quercetin-3-0- $\beta$-D-rhamnoside & $\mathrm{OH}$ & $\mathrm{OH}$ & $\mathrm{H}$ & O-rhamnoside & $\mathrm{OH}$ \\
\hline Quercetin-3-0-glucoside & $\mathrm{OH}$ & $\mathrm{OH}$ & $\mathrm{H}$ & O-glucoside & $\mathrm{OH}$ \\
\hline Quercetin-3-0-rutinoside & $\mathrm{OH}$ & $\mathrm{OH}$ & $\mathrm{H}$ & 0-rutinoside & $\mathrm{OH}$ \\
\hline $\begin{array}{l}\text { Quercetin-3-0-(4-0-trans-caffeoyl)- } \alpha \text {-L-rhamno- } \\
\text { pyranosyl-(1-6)- } \beta \text {-D-galactopyranoside }\end{array}$ & $\mathrm{OH}$ & $\mathrm{OH}$ & $\mathrm{H}$ & $\begin{array}{l}\text { [4-0-trans-caffeoyl- } \alpha \text {-L-rhamno-pyranosyl- } \\
(1-6)-\beta \text {-D-galactopyranoside] }\end{array}$ & $\mathrm{OH}$ \\
\hline $\begin{array}{l}\text { Kaempferol-3-0-rhamnoside } \\
\text { Flavones }\end{array}$ & $\mathrm{H}$ & $\mathrm{OH}$ & $\mathrm{H}$ & O-rhamnoside & $\mathrm{OH}$ \\
\hline Apigenin & $\mathrm{H}$ & $\mathrm{OH}$ & $\mathrm{H}$ & $\mathrm{H}$ & $\mathrm{OH}$ \\
\hline Apigenin-4'-O- $\beta$-D-glycoside & $\mathrm{H}$ & O-glycoside & $\mathrm{H}$ & $\mathrm{H}$ & $\mathrm{OH}$ \\
\hline Apigenin-7-0- $\beta$-D-glycoside & $\mathrm{H}$ & $\mathrm{OH}$ & $\mathrm{H}$ & $\mathrm{H}$ & O-glycoside \\
\hline Luteolin & $\mathrm{OH}$ & $\mathrm{OH}$ & $\mathrm{H}$ & $\mathrm{H}$ & $\mathrm{OH}$ \\
\hline Luteolin-7-0- $\beta$-D-glycoside & $\mathrm{OH}$ & $\mathrm{OH}$ & $\mathrm{H}$ & $\mathrm{OH}$ & O-glycoside \\
\hline
\end{tabular}

Table 6: List of natural sweeteners

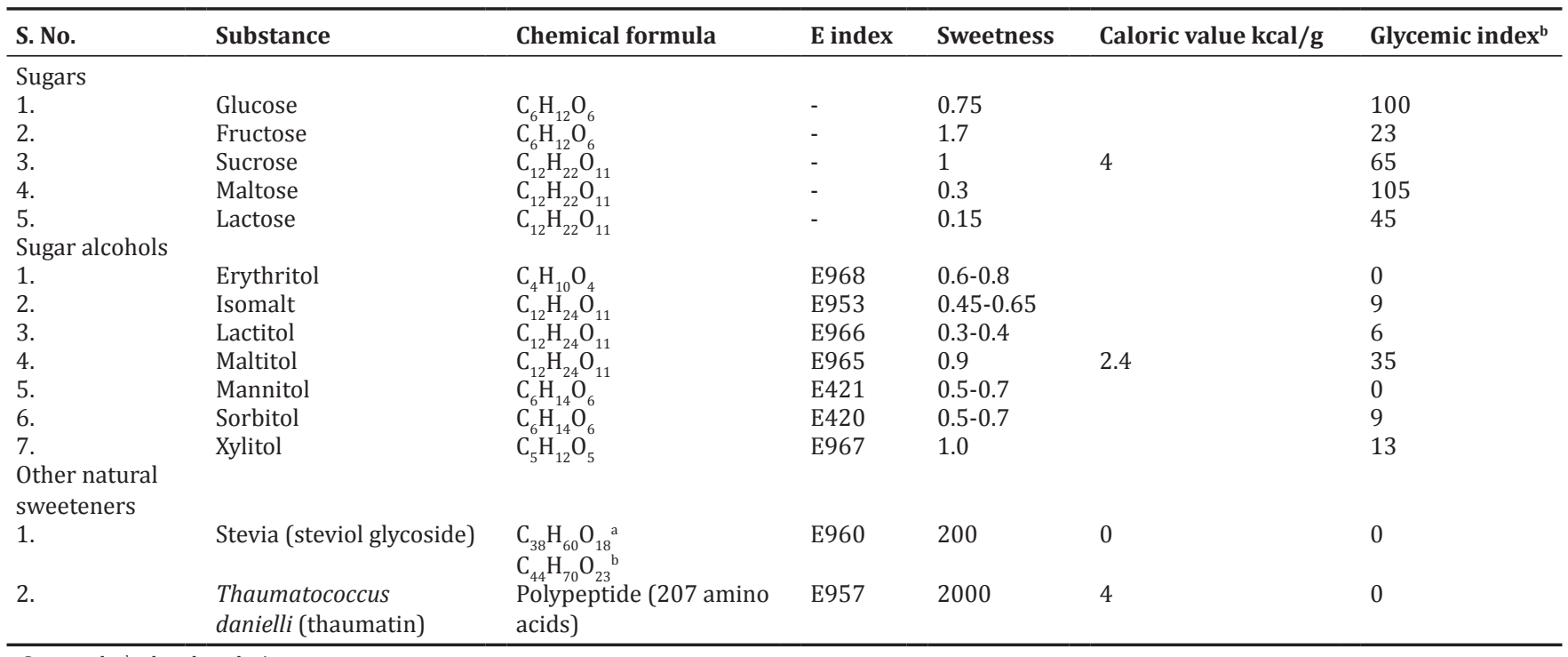

aStevioside, ${ }^{\text {brebaudioside A }}$

Table 7: Ethnomedical uses of stevia

\begin{tabular}{ll}
\hline Country & Ethnomedical uses \\
\hline United States & $\begin{array}{l}\text { Diabetes, candida, hyperglycemia, hypertension, } \\
\text { vasodilator, infections }\end{array}$ \\
South America & $\begin{array}{l}\text { Hypertension, diabetes, obesity, infections } \\
\text { Bepression, urinary insufficiency, tonic, } \\
\text { hyperglycemia, diabetes, infections, sweet } \\
\text { cravings, obesity, hypertension, cavities, wounds, } \\
\text { fatigue } \\
\text { Daraguay }\end{array}$ \\
\hline
\end{tabular}

biotechnological techniques and other toxicity studies are essential for the establishment of biomedical potentials of Stevia. Being a natural product, with virtually calorie free status causing less harm, Stevia benefits several health conditions reflecting its bright future with other medicinal values apart from its use as a sweetener.
Table 8: Commercially available stevia products in the USA market

\begin{tabular}{|c|c|c|}
\hline Product & $\begin{array}{l}\text { Dosage } \\
\text { form }\end{array}$ & Manufacturer \\
\hline Stevia tablets & Tablets & Stevia Now (Shrub Oak, USA) \\
\hline $\begin{array}{l}\text { Stevia pure } \\
\text { powder extracts }\end{array}$ & $\begin{array}{l}\text { Powder } \\
\text { extract }\end{array}$ & Stevia Now (Shrub Oak, USA) \\
\hline $\begin{array}{l}\text { Stevia } \\
\text { dark liquid } \\
\text { concentrate }\end{array}$ & $\begin{array}{l}\text { Liquid } \\
\text { concentrate }\end{array}$ & Stevia Now (Shrub Oak, USA) \\
\hline Stevia & Crystals & Stevia LLC (Valley Forge, PA, USA) \\
\hline Stevia extracts & Powder & $\begin{array}{l}\text { Life extension foundation (FL, } \\
\text { USA) }\end{array}$ \\
\hline $\begin{array}{l}\text { Stevia liquid } \\
\text { extract }\end{array}$ & Liquid & $\begin{array}{l}\text { Barr Products, } \\
\text { Inc. (Downingtown, PA, USA) }\end{array}$ \\
\hline JAJA stevioside & Powder & $\begin{array}{l}\text { JAJ Group, Inc. (Jacksonville, FL, } \\
\text { USA) }\end{array}$ \\
\hline
\end{tabular}




\section{AUTHORS CONTRIBUTIONS}

All the author have contributed equally.

\section{CONFLICTS OF INTERESTS}

All authors have none to declare.

\section{REFERENCES}

1. Ramesh K, Singh V, Megeji NW. Cultivation of stevia (Stevia rebaudiana Bertoni): A comprehensive review. Adv Agron 2006;89:137-77.

2. Kinghorn AD, editor. Overview. Stevia, the Genus of Stevia, Medicinal and Aromatic Plants Industrial Profiles. London: Taylor and Francis; 2002. p. 1-17.

3. Gupta R, Yadav V, Rastogi M. A review on importance of natural sweetener, a zero calorie plant-stevia-having medicinal and commercial importance. Int J Food Nutr Sci 2014;3:90-4.

4. Available from: http://www.internationalsteviacouncil.org. [Last accessed on 2017 Apr 19].

5. Tavarini S, Angelini LG. Stevia rebaudiana bertoni as a source of bioactive compounds: The effect of harvest time, experimental site and crop age on steviol glycoside content and antioxidant properties. J Sci Food Agric 2013;93:2121-9.

6. Wölwer-Rieck U. The leaves of Stevia rebaudiana (Bertoni), their constituents and the analyses thereof: A review. J Agric Food Chem 2012;60:886-95.

7. Brandle JE, Starratt AN, Gijzen M. Stevia rebaudiana: Its agricultural, biological, and chemical properties. Can J Plant Sci 1998:78:527-36.

8. Ohta M, Sasa S, Inoue A, Tamai T, Fujita I, Morita K, et al. Characterization of novel steviol glycosides from leaves of Stevia rebaudiana morita. J Appl Glycosci 2010;57:199-209.

9. Charturvedula VS, Clos JF, Rhea J, Milanowski D, Mocek U, DuBois GE, et al. Minor diterpenoid glycosides from the leaves of Stevia rebaudiana. Phytochem Lett 2011;6:175-8

10. Chaturvedula VS, Rhea J, Milanowski D, Mocek U, Prakash I. Two minor diterpene glycosides from the leaves of Stevia rebaudiana. Nat Prod Commun 2011;6:175-8.

11. Chaturvedula VS, Prakash I. Structures of the novel diterpene glycosides from Stevia rebaudiana. Carbohydr Res 2011;346:1057-60.

12. Charturvedula VS, Upreti M, Prakash I. Structures of the novel a-glycosyl linked diterpene glycoside from Stevia rebaudiana. Carbohydr Res 2011;346:2034-8

13. Kumari M, Chandra S. Phytochemical studies and estimation of major steviol glycosides in varied parts of Stevia rebaudiana. Int J Pharm Pharm Sci 2015;7:62-5.

14. Charturvedula VS, Upreti M, Prakash I. Diterpene glycosides from Stevia rebaudiana. Molecules 2011;16:3552-62.

15. Zimmermann BF. Tandem mass spectrometric fragmentation patterns of known and new steviol glycosides with structure proposals. Rapid Commun Mass Spectrom 2011;25:1575-82

16. Kim SH, Dubois GF. Natural high potency sweeteners. In: Marie S, Piggott JR, editors. Handbook of Sweeteners. Glasgow, New York: Blacki Avi; 1991.

17. Prakash I, Dubois GE, Clos JF, Wilkens KL, Fosdick LE. Development of rebiana, a natural, non-caloric sweetener. Food Chem Toxicol 2008;46 Suppl 7:S75-82

18. McGarvey BD, Attygalle AB, Starratt AN, Xiang B, Schroeder FC, Brandle JE, et al. New non-glycosidic diterpenes from the leaves of Stevia rebaudiana. J Nat Prod 2003;66:1395-8.

19. Supriyadi S, Siswandono S, Yuwono M. Method development and validation for the simultaneous determination Of stevioside, rebaudioside-A, rebaudioside C and dulcoside a contained in Stevia rebaudiana Bertoni using HPLC-ELSD. Int J Pharm Pharm Sci 2016;8:1-5.

20. Oshima Y, Saito J, Hikino H. Sterebin-E, sterebin-F, sterebin-G and sterebin-H, diterpenoids of Stevia rebaudiana leaves. Phytochemistry 1988;27:624-6.

21. Ibrahim NA, El-Gengaihi S, Motawe H, Riad SA. Phytochemical and biological investigation of Stevia rebaudiana Bertoni; 1-labdane-type diterpene. Eur Food Res Technol 2007;224:483-8.

22. Sholichin M, Yamasaki K, Miyama R, Yahara SS, Tanaka O. Labdanetype diterpenes from Stevia rebaudiana. Phytochemistry 1980;19:326.

23. Kim IS, Yang M, Lee OH, Kang SN. The antioxidant activity and the bioactive compound content of Stevia rebaudiana water extracts. LWT Food Sci Technol 2011;44:1328-32.

24. Karaköse H, Jaiswal R, Kuhnert N. Characterization and quantification of hydroxycinnamate derivatives in Stevia rebaudiana leaves by LC-MSn. J Agric Food Chem 2011;59:10143-50.

25. Cacciola F, Delmonte P, Jaworska K, Dugo P, Mondello L, Rader JI, et al. Employing ultra-high pressure liquid chromatography as the second dimension in a comprehensive two-dimensional system for analysis of Stevia rebaudiana extracts. J Chromatogr A 2011;1218:2012-8.

26. Ghanta S, Banerjee A, Poddar A, Chattopadhyay S. Oxidative DNA damage preventive activity and antioxidant potential of Stevia rebaudiana (Bertoni) bertoni, a natural sweetener. J Agric Food Chem 2007;55:10962-7.

27. Tadhani MB, Patel VH, Subhash R. In vitro antioxidant activities of Stevia rebaudiana leaves and callus. J Food Compos Anal 2007;20:323-9.

28. Li J, Jiang H, Shi R. A new acylated quercetin glycoside from the leaves of Stevia rebaudiana bertoni. Nat Prod Res 2009;23:1378-83.

29. Michalik A, Hollinshead J, Jones L, Fleet GW, Yu CY, Hu XG, et al. Steviamine, a new indolizidine alkaloid from Stevia rebaudiana. Phytochem Lett 2010;3:136-8.

30. Winchester BG. Iminosugars: From botanical curiosities to licensed drugs. Tetrahedron 2009;20:645-51.

31. EFSA. Scientific opinion on the safety and efficacy of thaumatin for all animal species. EFSA J 2011;9:2354-64.

32. Grembecka M. Sugar alcohols their role in the modern world of sweeteners a review. Eur Food Res Technol 2015;241:1-14

33. Livesey G. Health potential of polyols as sugar replacers, with emphasis on low-glycaemic properties. Nutr Res Rev 2003;16:163-91.

34. Nurkhasanah, Minangsari DN, Yulianny VA. The combination of rosella (Hibiscus sabdariffa, L) and stevia (Stevia rebaudiana) extracts increase the antioxidant activity and stability. Int J Pharm Pharm Sci 2016;8:411-2.

35. Taylor L. The Healing Power of Natural Herbs. Garden City Park, New York: Square One Publishers, Inc.; 2005. 